\title{
Dirty Hands Make Dirty Leaders?! The Effects of Touching Dirty Objects on Rewarding Unethical Subordinates as a Function of a Leader's Self-Interest
}

\author{
Florien M. Cramwinckel - David De Cremer • \\ Marius van Dijke
}

Received: 23 May 2012/Accepted: 11 June 2012/Published online: 28 June 2012

(C) The Author(s) 2012. This article is published with open access at Springerlink.com

\begin{abstract}
We studied the role of social dynamics in moral decision-making and behavior by investigating how physical sensations of dirtiness versus cleanliness influence moral behavior in leader-subordinate relationships, and whether a leader's self-interest functions as a boundary condition to this effect. A pilot study $(N=78)$ revealed that when participants imagined rewarding (vs. punishing) unethical behavior of a subordinate, they felt more dirty. Our main experiment $(N=96)$ showed that directly manipulating dirtiness by allowing leaders to touch a dirty object (fake poop) led to more positive evaluations of, and higher bonuses for, unethical subordinates than touching a clean object (hygienic hand wipe). This effect, however, only emerged when the subordinate's unethical behavior did not serve the leader's own interest. Hence, subtle cues such as bodily sensations can shape moral decision-making and behavior in leader-subordinate relationships, but selfinterest, as a core characteristic of interdependence, can override the influence of such cues on the leader's moral behavior.
\end{abstract}

Keywords Ethical leadership - Leader · Physical cleansing . Physical cues - Rewarding behavior . Subordinate

F. M. Cramwinckel ( $\square)$

Faculty of Social Sciences, Department of Social Psychology, Utrecht University, Heidelberglaan 1, Utrecht, The Netherlands e-mail: F.M.Cramwinckel@uu.nl

D. De Cremer · M. van Dijke

Rotterdam School of Management, Erasmus University,

Rotterdam, The Netherlands

D. De Cremer

London Business School, London, UK

\section{Introduction}

Ethical leaders are expected not only to behave ethically themselves, but also to promote ethical behavior in their subordinates, for example, by rewarding ethical behavior and punishing unethical behavior (Treviño et al. 2000, 2003; Treviño and Brown 2005). In reality, however, this does not always happen; leaders sometimes even reward unethical behavior. In the present studies, we want to examine the notion that the ethical behavior of leaders can be influenced by subtle cues related to immorality (i.e., dirty objects in this case), but also by other conflicting motives such as selfinterest. By means of this approach, we hope to shed more light on the processes that underlie leaders' decisions to reward ethical transgressions conducted by followers. Furthermore, we examine whether research on the moral-purity metaphor (e.g., Zhong and Liljenquist 2006; Zhong et al. 2010b) can be extended to the domain of interdependent social relationships, specifically the leader-subordinate relationship, and also identify a theoretically relevant boundary condition to this effect in such relationships.

The essence of who we are as social, moral, and also self-interested beings is to a large extent shaped by how we relate to others (Baumeister and Leary 1995; Brewer 2004; Rai and Fiske 2011). Many of our relationships with others involve the exchange of both tangible (e.g., money) and intangible (e.g., love, friendship, and support) goods, and can thus be considered as highly socially interdependent (Van Lange et al. 2007). The notion of social interdependence holds that people's decisions and actions influence their interaction partners' outcomes (and vice versa). Highly interdependent social relationships are, therefore, by definition, characterized by the presence of different motives. Specifically, when relationships are socially interdependent, both the motive to do the right thing 
(i.e., morality) and the motive to promote one's welfare (i.e., self-interest) can be salient. Both motives (morality and self-interest) should thus be able to influence people's ethical behaviors and decisions.

One important case of an interdependent relationship is the leader-subordinate relationship. The relationships between leaders and subordinates are colored by concerns about fairness and morality, but also by self-interest (Van den Bos and Lind 2002), which make this specific type of relationship a complex one. Indeed, the complexity of relationships between followers and subordinates is demonstrated by the observation that leaders are simultaneously responsible to maintain their own and their subordinates' morality, while also expected to be personally successful and acquire desired outcomes. Outcomes for which they, in turn, often depend to a large extent on the actions of their subordinates (Kramer 1996; Treviño et al. 2000). Because of this inherent complexity, the influence that the different motives of morality and selfinterest exert on ethical behavior is likely to depend on the extent to which these motives conflict or align with each other. This potential conflict becomes very clear in situations-which are also the focus of the present researchwhere leaders have to decide on allocating valuable outcomes such as the decision to provide or withhold (financial) bonuses for (un)ethical subordinate behavior. In fact, when morality and self-interest motives conflict under such circumstances, it could happen that leaders reward subordinates who have acted unethically.

In the present paper, we argue that the degree to which moral transgressions of subordinates are rewarded depends on how leaders interpret and judge these transgressions (i.e., referred to as their frame of reference in the present paper). For example, when a subordinate performs exceptionally well, a leader would probably evaluate this subordinate very positively, and could use this positive evaluation as a motivation to reward this subordinate. It is, however, also possible that this performance is due to unethical behavior such as cheating, stealing, or lying. In that case, a leader would be expected to have a less positive evaluation of this subordinate and to use this negative evaluation as a motivation to punish this subordinate. So the decision to reward or punish a high-performing subordinate can depend on whether a leader positively or negatively evaluates this (transgressing) subordinate.

Interestingly, actual ethical or unethical behavior of a subordinate may not be the only factor that influences a leader's frame of reference. Recent research has revealed convincing evidence that people's frame of reference and subsequent behavior can be influenced not only by subtle cues related to morality, such as bodily sensations of dirtiness or cleanliness (Zhong et al. 2010b; Lee and Schwarz 2010; Liljenquist et al. 2010; Schnall et al. 2008a), but also by opposing cues, such as self-interest (Aquino et al. 2009)—although this has not been examined yet in the context of leader-subordinate relationships.

Bodily sensations are heavily intertwined with moral judgment and behavior as illustrated by research on the metaphorical relationship between physical and moral purity (Zhong and Liljenquist 2006; Zhong et al. 2010b). The physical experience of cleanliness, for instance, leads people to render harsher judgments of hypothetical moral transgressions relative to the experience of dirtiness (Zhong et al. 2010b). Bodily sensations can also influence actual moral behavior. Wearing sunglasses, and thus experiencing a subjective sense of darkness, can, for example, increase subsequent unethical behavior (Zhong et al. 2010a). Clean smells, on the other hand, can promote virtuous behavior (Liljenquist et al. 2010). Physical sensations related to cleanliness thus seem to increase the influence of morality concerns on judgment and behavior, while physical sensations of dirtiness seem to do the opposite.

Although prior studies have deepened our insights into the relationship between morality and purity, to our knowledge, however, they focus primarily on the effect of bodily sensations on behavior that has little or no direct relevance to other people-in our case, others being subordinates (see Zhong and Liljenquist 2006, for an exception). In other words, these prior studies have investigated primarily the influence of bodily sensations on people's moral judgments and decisions outside the context of interdependent social relationships. As we noted earlier, this perspective makes it clear that the influence of bodily sensations on people's judgments and behaviors has not been applied yet to the context of the leader-subordinate relationship. This is regretful because particularly in research related to morality, the social context is very important since most (im)moral judgments and behaviors have consequences for (interdependent) others. In the present paper, we, therefore, focus on how a leader's moral judgment and behavior regarding interdependent others (i.e., subordinates) vary as a function of morality and selfinterest motives which are, in turn, triggered by physical sensations of cleanliness or dirtiness.

Building on the existing literature, we would expect that unethical behavior of others will not be accepted when the leader experiences a physical sensation of cleanliness, since feeling clean has been shown to lead to harsher moral judgment (Zhong et al. 2010b). This suggests that when leaders feel clean they will judge subordinate's moral transgressions as less positive, and therefore will be less likely to reward the unethical behavior of the subordinate. In contrast, when the leader experiences a physical sensation of dirtiness, unethical behavior of subordinates will be judged as less severe and thus not necessarily negative, and 
this will lead to more rewarding behavior for moral transgressors.

However, it is important that this rather straightforward prediction may not always be valid in highly interdependent settings. Specifically, when social interdependence is high, leaders may also be affected by self-interest concerns, and then bodily sensations related to morality may exert less influence on their decision-making. Why? The existence of a conflict between self-interest and morality concerns is one of the key aspects of interdependent relationships (Rusbult and Van Lange 2003), and morality and self-interest are suggested to be two frames of reference that stand diametrically opposed to one another (Schwartz 1992; Grouzet et al. 2005; Aquino et al. 2009). When two opposing frames of reference are activated simultaneously, an aversive state of mind arises, and to resolve this, one of the two frames will become deactivated. Important, however, to this line of reasoning is that the literature suggests that when selfinterest is pitted against another frame of reference (morality in this case), self-interest is likely to prevail (Moore and Loewenstein 2004). This means that when both morality and self-interest motives play a role, the influence of morality motives on behavior will probably be undermined by selfinterest. Self-interest may thus be a relevant boundary condition to the effectiveness of bodily sensations in shaping a leader's moral judgments and decisions.

The above reasoning leads us to predict that leaders experiencing physical dirtiness (vs. cleanliness) will only lead to more positive evaluations of, and higher rewards for, an unethical subordinate when the leader does not benefit from the transgression. When there is no selfinterest, there will be no conflict between morality and selfinterest, and cues related to morality (such as dirtiness) can still have an influence on behavior. On the other hand, when the leader does benefit from the subordinate's transgression, self-interest will override the influence that morality cues exert on the leader's moral decision-making. In this case, there will be a conflict between morality and self-interest, and we expect self-interest to override the effect of morality, thereby eliminating the influence that cues related to morality (such as dirtiness) have on judgment and behavior.

Because prior research on bodily sensations and morality has neglected the socially interdependent context of many of our relationships, it is important to first examine the relevance of the cleanliness-dirtiness distinction in the context of the leader-subordinate relationship. For this purpose, we first conducted a pilot study. In this pilot study, we examined the untested prediction that rewarding (vs. punishing) a subordinate's moral transgression leads to more feelings of dirtiness in the target person (i.e., the evaluating person). This pilot test is thus designed to provide initial evidence that it is valid to investigate the (im)moral-(im)purity metaphor also in the domain of social relationships. In our main study, we will examine whether directly manipulating the leader's dirtiness (by touching a dirty vs. clean object) will lead to more positive evaluations of, and higher rewards for, subordinates engaging in moral transgressions as a function of the leader's selfinterest (see Fig. 1 for the proposed model).

\section{Pilot Study}

Seventy-eight participants $\left(M_{\text {age }}=24.0,46.9 \%\right.$ female $)$ were asked to participate in a vignette study. They were asked to read one of two scenarios and to imagine how they would feel if they would have experienced this situation. In the reward condition, participants imagined rewarding unethical behavior of a subordinate by allocating a financial bonus, while in the punishment condition, participants imagined punishing the unethical behavior of a subordinate by withholding a financial bonus. Participants read the following vignette. In the punishment condition, the underlined word ("not") was added.

Imagine that you are the leader of a team of two subordinates, Robin and Sanne. The task of Robin and Sanne is to independently complete several tasks as quickly as possible, while scoring as high as possible. The better your subordinates perform, the better your team compares to other teams, which is important to you as a leader. After completing the tasks, results showed that both Robin and Sanne performed very well. Robin performed exceptionally well, he managed to get the highest score in the least amount of time and therefore your team (under your supervision) performed much better than all the other teams.

While walking across the hallway, you accidentally hear a conversation between Robin and Sanne where Robin explains that he achieved his high score because he cheated: $\mathrm{He}$ accidentally acquired the answers for the task and was therefore able to quickly answer all the questions on the task correctly.

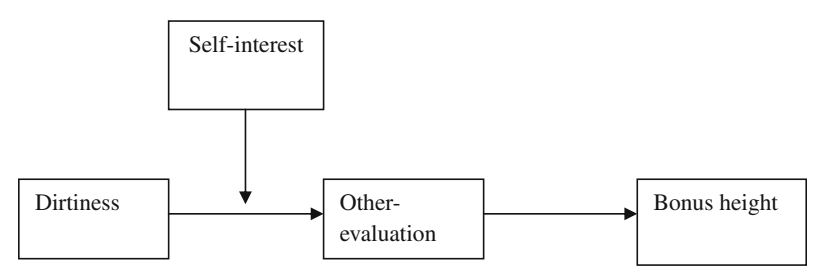

Fig. 1 Conceptual model 
There is a financial bonus reserved for the highest performing subordinate. Because of Robin's performance, and the way that he achieved this performance, you decide (not) to allocate this financial bonus to Robin.

After reading the vignette, participants were asked how clean, dirty, filthy, fresh, pure, stained, and ethical they would feel after allocating/withholding a financial bonus to Robin. Finally, participants answered three manipulation check items to check whether they had understood the vignette correctly. These items were "according to this scenario: did Robin achieve the highest score (yes/no), which of your subordinates cheated (Robin/ Sanne), was there a bonus allocated to Robin (yes/no)". Afterward, participants were thanked for their participation.

\section{Results and Discussion Pilot Study}

All participants indicated that they understood the scenario correctly by answering all three manipulation check items correctly. As expected, participants in the reward condition felt more ethical than in the punishment condition. More importantly, participants in the reward condition felt more dirty, filthy, and stained, but less clean, fresh, and pure (all p's <.001) than participants in the punishment condition. See Table 1 for ANOVA test statistics and cell means.

This study thus showed that rewarding unethical behavior of others is related to the experience of dirtiness, while punishing unethical behavior of others is related to the experience of cleanliness. It thereby provides initial support for our notion that the (im)moral-(im)purity metaphor is also relevant in the domain of interdependent relationships such as the leader-subordinate relationship.

\section{Main Study}

In our main study, we examined whether manipulated dirtiness (relative to cleanliness) leads to higher rewards for subordinates engaging in moral transgressions, but only so when the leader has no self-interest in the transgression. Moreover, we expect this relationship to be mediated by how the leader evaluates the transgressor. Previous research (Zhong et al. 2010b) showed that when people feel dirty (after reading and copying a text about being dirty or clean) rather than clean, people feel less moral themselves, which makes them more lenient about possible moral transgressions since they do not feel morally superior. In the context of highly interdependent social relationships, we expect that the underlying process is not how people evaluate themselves, but how they evaluate the interdependent other. We expect that when people feel dirty, they evaluate moral transgressions of others more leniently since they do not feel morally superior to the other person. We therefore expect that participants in the dirty condition will evaluate the transgressing participant more positively than participants in the clean condition will. To manipulate physical sensations, participants put in the leadership position evaluated either a dirty (fake poop) or clean (antiseptic hand wipes) object before deciding how much to reward the cheating subordinate.

\section{Method}

\section{Participants}

Ninety-three undergraduate students $\left(M_{\text {age }}=20.40\right.$, $35.4 \%$ female) participated in exchange for course credits and were randomly assigned to one of the four conditions of a 2 (self-interest: high vs. low) $\times 2$ (dirtiness: clean vs. dirty) between-subjects design.

Table 1 Descriptives of cleanliness/dirtiness for reward and punishment conditions in pilot study

\begin{tabular}{|c|c|c|c|c|c|c|c|c|c|}
\hline & \multicolumn{3}{|c|}{ Reward condition } & \multicolumn{3}{|c|}{ Punishment condition } & \multirow{2}{*}{$\begin{array}{l}\text { Multivariate } \\
F\end{array}$} & \multicolumn{2}{|l|}{ Univariate } \\
\hline & $N$ & $M$ & SD & $N$ & $M$ & SD & & $F$ & $\eta^{2}$ \\
\hline To what extent would you feel...? & 37 & & & 41 & & & $16.18 * * *$ & & \\
\hline Clean & & 2.73 & 1.59 & & 5.51 & 1.31 & & $71.73 * * *$ & .09 \\
\hline Fresh & & 2.95 & 1.53 & & 5.37 & 1.32 & & $56.42 * * *$ & .07 \\
\hline Pure & & 2.41 & 1.42 & & 5.63 & 1.28 & & $111.31 * * *$ & .12 \\
\hline Ethical & & 2.35 & 1.60 & & 5.88 & 1.47 & & $102.81 * *$ & .13 \\
\hline Dirty & & 5.03 & 1.68 & & 2.32 & 1.39 & & $61.03 * * *$ & .11 \\
\hline Filthy & & 4.76 & 1.82 & & 2.27 & 1.36 & & $47.46 * * *$ & .10 \\
\hline Stained & & 4.84 & 1.63 & & 3.32 & 1.92 & & $14.13 * * *$ & .03 \\
\hline
\end{tabular}

$* p<.05 ; * * p<.01 ; * * * p<.001$ 


\section{Procedure}

Participants were placed behind a computer in separate cubicles. All communication was done via the computer which was supposedly connected to a general server. They were asked to respond to some background questions (e.g., age, gender) and to fill out some personality questionnaires that were said to be related to leadership qualities, e.g., Sense of Power (eight items, e.g., "I think I have a great deal of power in my relationships with others"; Anderson et al. 2005) and Achievement Motivation (14 items, e.g., "I would be a good leader"; Cassidy and Lynn 1989, as used in Maner and Mead 2010). Afterward, they learned that they would be working together with two other participants in the lab, and that the most suitable candidate of the three (ostensibly based on their scores on the personality questionnaires) would be assigned the leader role (see also Rus et al. 2012; DeWall et al. 2011). In reality, all participants were assigned this role and were coupled with two bogus subordinates.

Participants then received information about their leader role and what it entailed. They learned that they had to coordinate the group tasks, check and evaluate the answers of their subordinates, and decide whether or not to allocate a bonus to their subordinates. They were also told that they would receive an overview of the performance of their subordinates, and it would be up to them to approve these performances or not. They also learned about the tasks that the subordinates were required to undertake. Specifically, participants learned that the subordinates were to solve difficult math problems as fast as possible, while also being as accurate as possible.

\section{Self-Interest Manipulation}

Participants then learned that they could be rewarded a leader-bonus either regardless of the performance of their subordinates (no self-interest condition) or only if their subordinates performed well (self-interest condition).

\section{Cleanliness/Dirtiness Manipulation}

Before engaging in their specific leader tasks (evaluating and checking the performance of their subordinates and providing/withholding a financial bonus), participants were asked to complete an unrelated task, while their subordinates were ostensibly solving these difficult math problems. Participants were redirected to a screen indicating that they were now decoupled from the other two (bogus) participants, and to make this clear, they ended up in another task environment that had a completely different lay-out (different colors, different fonts, different set-up, etc.) than the one they were previously in. Participants learned that they were now participating in a study about "human judgment and expression," which consisted of two parts. In the first part they were asked to copy a statement onto a piece of paper so their handwriting could be judged and evaluated on notions of "personal expression." The tobe-copied statement was either a short text about being physically clean (clean condition) or about being physically dirty (dirty condition. See Zhong et al. 2010b for the exact texts). Hereafter, participants were asked to engage in the second part of the study, which was a product evaluation. They were asked touch, smell, and evaluate a dirty (fake poop) or clean (hygienic cleansing wipe) product and answer several questions about this product. These questions were how "handy," "pretty," "functional," "nice," "clean," “dirty," "useless," “weird," “funny," and "realistic" they thought this product was $(1=$ not at all, $7=$ completely). They also answered to what extent they would like to have this product, if they thought this product smelled nice, if they thought this product felt clean, if they would buy this product in a store and if they felt dirty after touching this product $(1=$ not at all, $7=$ completely $)$. They also indicated how much Euro they would like to pay for this product. Two of these questions (how "clean" and "dirty" they thought the product was) which were embedded in the other questions served as manipulation checks. Afterward, participants were thanked for their participation and could close the survey.

After they closed the survey, the participants were redirected to the main experiment and were re-coupled with the two (bogus) subordinates. Participants were notified that they had to wait until their subordinates had finished the math task before they could proceed. They saw a screen where they ostensibly could follow the performance of their subordinates in real-time. After their subordinates had completed the task, participants received information about their team members' performance. This information showed that team member A had performed exceptionally well on the task: this subordinate had answered 20 out of 20 questions correctly in about three minutes, while the average performance of all participants in this study was 14 correct questions in $15 \mathrm{~min}$ (all information provided was fictional).

Subsequently, they received some information about how their subordinates had worked on their task, by reading a bogus transcript of communication between team member A and B. Participants saw an MSN-messenger screen on which team member A said that he/she had achieved this high performance because there was a note with the correct answers in his/her cubicle, which was probably left there by a previous participant. Team member A further said that he/she had used this note to complete the task very quickly. So the participants learned that their extremely high-performing subordinate had cheated on the task. 
Then, the dependent variables were solicited. First, team member A's evaluation $(\alpha=.85)$ was measured by asking participants to what extent they "thought member A did a good job" and "were satisfied with the work of member A" $(1=$ not at all, $7=$ very much so $)$.

Participants were then asked to assign a bonus between 0 and 10 Euros to team member A. Subsequently, the apparent evaluation of team member B was skipped, ostensibly due to a computer error. Finally, participants were thanked, debriefed, and dismissed.

\section{Results and Discussion}

\section{Manipulation Checks}

A $2 \times 2$ MANOVA on clean and dirty evaluations showed a significant effect of dirtiness, Wilks' lambda $F(2$, $93)=61.60, p<.001$. Univariate $F$ tests showed that participants evaluated the fake poop as dirtier $(M=5.27$ vs. $1.94, \mathrm{SD}=0.25$ vs. 0.25$), F(1,94)=87.85, p<.001$, $\eta^{2}=.48$, and less clean than $(M=2.20$ vs. 5.75, $\mathrm{SD}=.0 .23 \quad$ vs. 0.23$), \quad F(1,94)=124.42, \quad p<.001$, $\eta^{2}=.56$ the antiseptic wipe. The main effect of selfinterest and the self-interest $x$ dirtiness interaction was not significant.

\section{Bonus}

A $2 \times 2$ ANOVA on the bonus given to the cheating subordinate revealed a significant main effect of dirtiness, $F(1,89)=4.10, p=.046, \eta^{2}=.02$, showing that dirty participants allocated higher bonuses to the transgressing subordinate than the clean participants. This main effect was qualified by a significant self-interest $\times$ dirtiness interaction, $F(1,89)=5.09, p=.027, \eta^{2}=.03$ (see Fig. 2). Post hoc tests showed that when self-interest was low, dirty participants allocated a higher bonus to the transgressing subordinate than the clean participants $(M=3.68$ vs. $1.39, \mathrm{SD}=3.21$ and 1.95$)$, Tukey's HSD, $p=.016$. When self-interest was high, however, bonus height did not differ between dirty and clean $(M=2.48$ and 2.60, $\mathrm{SD}=1.89$ and 2.83) participants, Tukey's HSD, $p=.998$ (see Fig. 2).

\section{Mediation}

We subsequently tested our hypothesis that the influence of the dirtiness $\times$ self-interest interaction on bonus height was mediated by positive evaluations by means of mediated moderation analysis (Preacher et al. 2007). This analysis indicated, first of all, that the dirtiness $\times$ selfinterest interaction significantly influenced the positive

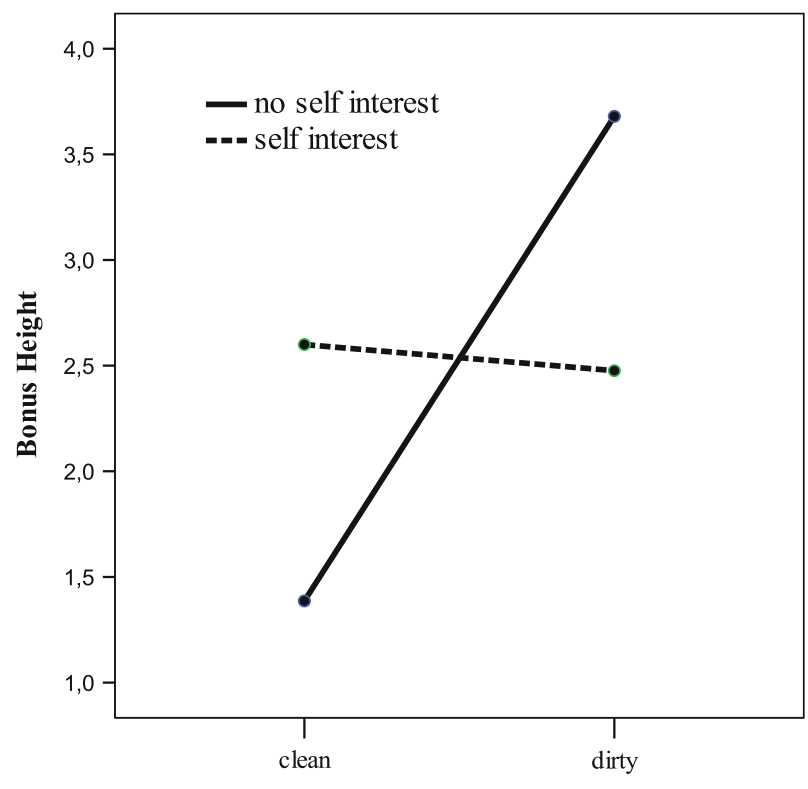

Fig. 2 Bonus height for dirtiness $\times$ self-interest interaction in main experiment

evaluation of the transgressing subordinate $(\beta=-21$, $t=-2.05, p=.043)$. Furthermore, this evaluation significantly influenced bonus height $(\beta=1.58, t=11.96$, $p<.001)$. We relied on 5,000 bootstrap resamples to obtain estimates for the indirect effect of dirtiness on bonus height via positive evaluation (as a function of self-interest). Dirtiness (relative to cleanliness) led to higher bonuses, via positive evaluation, when no self-interest was involved (indirect effect $=.45,95 \%$ CI .08-.91). However, dirtiness did not influence bonus height via positive evaluation when self-interest was high (indirect effect $=$ $-.20,95 \% \mathrm{CI}-.67$ to .31$)$.

\section{General Discussion}

In a pilot study, we demonstrated that the moral-purity metaphor might be extended to the domain of highly interdependent, social relations. Our main study shows that bodily sensations of dirtiness versus cleanliness influence whether leaders respond to moral transgressions of subordinates in terms of positive evaluations and subsequent rewarding behavior. Specifically, cheating on a task was more highly evaluated and, consequently, resulted in a higher reward when the leader had a dirty, relative to a clean, frame of reference. However, this effect was restricted to situations in which the moral transgression did not serve the leader's self-interest.

Although a number of studies on the relationship between bodily sensations and morality have appeared over 
the past few years (e.g., Eyal et al. 2008; Schnall et al. 2008a, b; Zhong et al. 2010a), this work has so far disregarded the notion that "all of the building blocks of human psychology (...) have been shaped by the demands of social interdependence" (Brewer 2004, p. 107). Recognizing this interdependent context shows that bodily sensations shape not only evaluations of abstract moral issues and dilemmas, but also concrete, morally valenced interpersonal behavior. Furthermore, the importance of interdependence is demonstrated by the fact that when interdependence is low (such as in previous research), there is a direct and positive relationship between physical sensations of cleanliness and moral judgment. On the other hand, when interdependence is high (as in our present research), the presence of different motives (e.g., morality vs. self-interest) changes the shape of this effect. The present findings suggest that when self-interest comes into play, the previously established direct and positive influence of cleanliness on moral judgment and behavior disappears.

Second, very little work has addressed a boundary condition to the physical-moral purity relationship. In fact, we know of only one: Schnall et al. (2008b) showed that the influence of disgust on moral judgment is especially strong for people who are chronically (i.e., dispositionally) sensitive to their bodily sensations. Taking into account the interdependent context in which moral behavior is enacted reveals self-interest as a theoretically relevant boundary condition to the effectiveness of embodied cognition in shaping evaluations of others and subsequent interpersonal behavior. A self-interest frame of reference can thus reduce the influence of a morality frame on moral behavior, and hence the influence of (im)purity on moral behavior.

Mirroring the neglect of moderating factors, there are also only few studies that have focused on mediators of the relationship between bodily sensations and moral behavior (see Caruso and Gino 2011; Zhong et al. 2010a for exceptions). Zhong et al. (2010a) show, for example, that physical cleansing leads to feelings of moral superiority which, in turn, lead to harsher judgments of morally ambiguous behaviors. However, in the context of interdependent relationships, evaluations of others are likely to be more important in shaping moral judgment and subsequent behavior than evaluations of the self. Our research is, to our knowledge, the first to investigate other-evaluation as a mediating mechanism for the relationship between physical (im)purity and moral behavior, and thereby the first to capture interdependent aspects of the process leading from bodily sensations to morality. We, therefore, suggest that interpersonal moral behavior in terms of rewarding or punishing others may be better explained by moral otherregulation (rather than by moral self-regulation (Zhong et al. 2009).
Ethical leaders are supposed to consciously manage their own, and others', morality by rewarding ethical and punishing unethical behavior (Treviño et al. 2000, 2003; Treviño and Brown 2005).This perspective implies that these leaders can control effectively and explicitly how they regulate their decisions with respect to the behaviors of others-as ethical leadership and acting as an ethical person in general is supposed to be a conscious and intentful decision (Jones 1991) However, research in behavioral business ethics is increasingly showing that the ethical capacities of people-and thus also our leaderscan be limited in implicit ways by our experiences and cues related to the social settings that we are part of (i.e., Bazerman and Tenbrunsel 2011; De Cremer and Tenbrunsel 2012; De Cremer et al. 2010). In fact, our research demonstrates how subtle cues in one's environment can influence how leaders judge and reward ethical transgressions of their followers. This is problematic since these subtle cues are difficult to control and are often not even noticed by those leading us. Despite the difficulty of being influenced by such subtle cues, it is important that we were also able to identify a relevant boundary condition to the influence of physical sensations on moral judgment and behavior: leader's self-interest. Specifically, our findings clearly suggest that if companies were to make sure that the interests of leaders align with the collective interest of the company, then the influence of subtle morality related cues such as in this case would no longer have such a detrimental effect on the leader's ethical decisions.

In conclusion, bodily sensations can shape moral interpersonal behaviors in interdependent settings. At the same time, when self-interest, as a key aspect of interdependence, is salient, bodily sensations are no longer relevant in shaping moral judgments and decisions.

Open Access This article is distributed under the terms of the Creative Commons Attribution License which permits any use, distribution, and reproduction in any medium, provided the original author(s) and the source are credited.

\section{References}

Anderson, C., John, O. P., \& Keltner, D. (2005). Journal of Personality, 80(2), 313-344.

Aquino, K., Freeman, D., Reed, A., I. I., Lim, V. K., \& Felps, W. (2009). Testing a social-cognitive model of moral behavior: The interactive influence of situations and moral identity centrality. Journal of Personality and Social Psychology, 97(1), 123-141.

Baumeister, R. F., \& Leary, M. R. (1995). The need to belong: Desire for interpersonal attachments as a fundamental human motivation. Psychological Bulletin, 117(3), 497-529.

Bazerman, M. H., \& Tenbrunsel, A. E. (2011). Blind spots: Why we fail to do what's right and what to do about it. Princeton, NJ: Princeton University Press. 
Brewer, M. B. (2004). Taking the social origins of human nature seriously: Toward a more imperialist social psychology. Personality and Social Psychology Review, 8(2), 107-113.

Caruso, E. M., \& Gino, F. (2011). Blind ethics: Closing one's eyes polarizes moral judgments and discourages dishonest behavior. Cognition, 118, 280-285.

Cassidy, T., \& Lynn, R. (1989). A multifactorial approach to achievement motivation: The development of a comprehensive measure. Journal of Occupational Psychology, 62, 301-312.

De Cremer, D., \& Tenbrunsel, A. E. (Eds.). (2012). Behavioral business ethics: Ideas on an emerging field. London: Taylor \& Francis.

De Cremer, D., Tenbrunsel, A. E., \& Van Dijke, M. (2010). Regulating ethical failures: Insights from psychology. Journal of Business Ethics, 95(SI 1), 1-6.

DeWall, C. N., Baumeister, R. F., Mead, N. L., \& Vohs, K. D. (2011). How leaders self-regulate their task performance: Evidence that power promotes diligence, depletion, and disdain. Journal of Personality and Social Psychology, 100(1), 47-65.

Eyal, T., Liberman, N., \& Trope, Y. (2008). Judging near and distant virtue and vice. Journal of Experimental Social Psychology, 44(4), 1204-1209.

Grouzet, F., Kasser, T., Ahuvia, A., Dols, J. M., Kim, Y., Lau, S., et al. (2005). The structure of goal contents across 15 cultures. Journal of Personality and Social Psychology, 89(5), 800-816.

Jones, T. M. (1991). Ethical decision making by individuals in organizations: An issue contingent model. Academy of Management Review, 16, 366-395.

Kramer, R. M. (1996). Divergent realities and convergent disappointments in the hierarchic relation: Trust and the intuitive auditor at work. In R. M. Kramer \& T. R. Tyler (Eds.), Trust in organizations: Frontiers of theory and research (pp. 216-245). Thousand Oaks, CA: Sage.

Lee, S. W., \& Schwarz, N. (2010). Dirty hands and dirty mouths: Embodiment of the moral-purity metaphor is specific to the motor modality involved in moral transgression. Psychological Science, 21, 1423-1425.

Liljenquist, K., Zhong, C.-B., \& Galinsky, A. D. (2010). The smell of virtue: Clean scents promote reciprocity and charity. Psychological Science, 21, 381-383.

Maner, J. K., \& Mead, N. L. (2010). The essential tension between leadership and power: When leaders sacrifice group goals for the sake of self-interest. Journal of Personality and Social Psychology, 90(3), 297-482.

Moore, D. A., \& Loewenstein, G. (2004). Self-interest, automaticity, and the psychology of conflict of interest. Social Justice Research, 17(2), 189-202.

Preacher, K. J., Rucker, D. D., \& Hayes, A. F. (2007). Assessing moderated mediation hypotheses: Theory, methods, and prescriptions. Multivariate Behavioural Research, 42, 185-227.

Rai, T. S., \& Fiske, A. P. (2011). Moral psychology is relationship regulation: Moral motives for unity, hierarchy, equality, and proportionality. Psychological Review, 118(1), 57-75.
Rus, D., Van Knippenberg, D., \& Wisse, B. (2012). Leader power and self-serving behavior: The moderating role of accountability. The Leadership Quarterly, 23(1), 13-26.

Rusbult, C. E., \& Van Lange, P. A. M. (2003). Interdependence, interaction, and relationships. Annual Review of Psychology, 54, 351-375.

Schnall, S., Benton, J., \& Harvey, S. (2008a). With a clean conscience: Cleanliness reduces the severity of moral judgments. Psychological Science, 19(12), 1219-1222.

Schnall, S., Haidt, J., Clore, G. J., \& Jordan, A. H. (2008b). Disgust as embodied moral judgment. Personality and Social Psychology Bulletin, 34(8), 1096-1109.

Schwartz, S. H. (1992). Universals in the content and structure of values: Theoretical advances and empirical tests in 20 countries. In M. P. Zanna (Ed.), Advances in experimental social psychology (Vol. 25, pp. 1-65). New York: Academic Press.

Treviño, L. K., \& Brown, M. (2005). The role of leaders in influencing unethical behavior in the workplace. In J. R. E. Kidwell \& C. L. Martin (Eds.), Managing organizational deviance (pp. 69-87). Thousand Oaks, CA: Sage.

Treviño, L. K., Brown, M., \& Hartman, L. P. (2003). A qualitative investigation of perceived executive ethical leadership: Perceptions from inside and outside the executive suite. Human Relations, 56(1), 5-37.

Treviño, L. K., Hartman, L. P., \& Brown, M. (2000). Moral person and moral manager: How executives develop a reputation for ethical leadership. California Management Review, 42(4), $128-142$.

Van den Bos, K., \& Lind, E. (2002). Uncertainty management by means of fairness judgments. Advances in Experimental Social Psychology, 34, 1-60. doi:10.1016/S0065-2601(02)80003-X

Van Lange, P. A. M., De Cremer, D., Van Dijk, E., \& Van Vugt, M. (2007). Self-interest and beyond: Basic principles of social interaction. In A. W. Kruglanski \& E. T. Higgins (Eds.), Social psychology: Handbook of basic principles (2nd ed., pp. 540-561). New York: Guilford.

Zhong, C. B., Bohns, V. K., \& Gino, F. (2010a). Good lamps are the best police: Darkness increases dishonesty and self-interested behavior. Psychological Science, 21(3), 311-314.

Zhong, C. B., \& Liljenquist, K. (2006). Washing away your sins: Threatened morality and physical cleansing. Science, 313, $1451-1452$.

Zhong, C., Liljenquist, K., \& Cain, D. M. (2009). Moral selfregulation. Licensing and compensation. In D. De Cremer (Ed.), Psychological perspectives on ethical behavior and decision making (pp. 75-119). Charlotte, NC: Information Age.

Zhong, C. B., Strejcek, B., \& Sivanathan, N. (2010b). A clean self can render harsh moral judgment. Journal of Experimental Social Psychology, 46, 859-862. 